\title{
Network meta-analysis of triazole, polyene, and echinocandin antifungal agents in invasive fungal infection prophylaxis in patients with hematological malignancies
}

Huilan Zeng ${ }^{1 \dagger}$, Zhuman $\mathrm{Wu}^{2+}$, Bing $\mathrm{Yu}^{1}$, Bo Wang ${ }^{1}$, Chengnian $\mathrm{Wu}^{1}$, Jie $\mathrm{Wu}^{1}$, Jing Lai ${ }^{1}$, Xiaoyan Gao and Jie Chen $^{3^{*}}$ (iD

\begin{abstract}
Background and aim: Triazole, polyene, and echinocandin antifungal agents are extensively used to treat invasive fungal infections (IFIs); however, the optimal prophylaxis option is not clear. This study aimed to determine the optimal agent against IFIs for patients with hematological malignancies.

Methods: Randomized controlled trials (RCTs) comparing the effectiveness of triazole, polyene, and echinocandin antifungal agents with each other or placebo for IFIs in patients with hematological malignancies were searched. This Bayesian network meta-analysis was performed for all agents.

Results: The network meta-analyses showed that all triazoles, amphotericin B, and caspofungin, but not micafungin, reduced IFIs. Posaconazole was superior to fluconazole [odds ratio (OR), 0.30; 95\% credible interval (Crl), 0.12-0.60], itraconazole (OR, 0.40; 95\% Crl, 0.15-0.85), and amphotericin B (OR, 4.97; 95\% Crl, 1.73-11.35). It also reduced allcause mortality compared with fluconazole $(\mathrm{OR}, 0.35$; $95 \% \mathrm{Crl}, 0.08-0.96)$ and itraconazole $(\mathrm{OR}, 0.33$; $95 \%$ Crl, 0.070.94), and reduced the risk of adverse events compared with fluconazole (OR, 0.02; 95\% Crl, 0.00-0.03), itraconazole $(\mathrm{OR}, 0.01 ; 95 \% \mathrm{Crl}, 0.00-0.02)$, posaconazole $(\mathrm{OR}, 0.02 ; 95 \% \mathrm{Crl}, 0.00-0.03)$, voriconazole $(\mathrm{OR}, 0.005 ; 95 \% \mathrm{Crl}, 0.00$ to $0.01)$, amphotericin $B(O R, 0.004 ; 95 \% \mathrm{Crl}, 0.00-0.01)$, and caspofungin $(\mathrm{OR}, 0.05 ; 95 \% \mathrm{Crl}, 0.00-0.42)$ despite no significant difference in the need for empirical treatment and the proportion of successful treatment.
\end{abstract}

Conclusions: Posaconazole might be an optimal prophylaxis agent because it reduced IFIs, all-cause mortality, and adverse events, despite no difference in the need for empirical treatment and the proportion of successful treatment.

Keywords: Echinocandin, Hematological malignancies, Invasive fungal infections, Network meta-analysis, Polyene, Prophylaxis, Triazole

\footnotetext{
* Correspondence: chenjiev001@163.com

${ }^{\dagger}$ Huilan Zeng and Zhuman Wu contributed equally to this work.

${ }^{3}$ Department of Urology Surgery, the First Affiliated Hospital of Jinan University, No.613 West Huangpu street, Guangzhou 510630, P. R. China

Full list of author information is available at the end of the article
}

C C The Author(s). 2021 Open Access This article is licensed under a Creative Commons Attribution 4.0 International License, which permits use, sharing, adaptation, distribution and reproduction in any medium or format, as long as you give appropriate credit to the original author(s) and the source, provide a link to the Creative Commons licence, and indicate if changes were made. The images or other third party material in this article are included in the article's Creative Commons. licence, unless indicated otherwise in a credit line to the material. If material is not included in the article's Creative Commons licence and your intended use is not permitted by statutory regulation or exceeds the permitted use, you will need to obtain permission directly from the copyright holder. To view a copy of this licence, visit http://creativecommons.org/licenses/by/4.0/ The Creative Commons Public Domain Dedication waiver (http://creativecommons.org/publicdomain/zero/1.0/) applies to the data made available in this article, unless otherwise stated in a credit line to the data. 


\section{Background}

Adult patients who were diagnosed with hematological malignancies, such as acute lymphoblastic leukemia [1], acute myeloid leukemia [2], or myelodysplastic syndrome, and then instructed to receive intensive chemotherapy for remission or hematopoietic stem cell transplantation (HSCT) were at high risk of developing invasive fungal infections (IFIs) [3, 4], especially Aspergillus- and Candida-related IFIs [5, 6]. IFIs contribute a lot to the morbidity and mortality in patients with hematological malignancies $[4,7]$ because the symptoms and signs are absent or nonspecific in the early stage $[8$, 9]. Thus, antifungal prophylaxis remains central to the containment of IFIs, making the early identification of IFIs difficult $[2,10,11]$.

Triazole, polyene, and echinocandin antifungal agents have been extensively applied to prevent and treat IFIs [3]. A large number of clinical trials have been performed to investigate the role of antifungal prophylaxis against IFIs [1, 2, 12-15]. Meanwhile, several metaanalyses have been performed to investigate the comparative efficacy and safety of the treatments $[3,4,16$, 17]. However, the previous meta-analyses were limited by some drawbacks such as insufficient number of eligible studies and treatments. Thus, which treatments should be preferably prescribed to patients who were at high risk of IFIs remained unclear.

The present Bayesian network meta-analysis combined direct and indirect evidence comparing the relative efficacy of all antifungal prophylaxis regimes to determine the optimal agents against IFIs among high-risk patients.

\section{Methods}

This systematic review and network meta-analysis was performed according to the methodology framework recommended by the Cochrane Collaboration, and all summarized results were reported in accordance with the Preferred Reporting Items for Systematic Reviews and Meta-Analyses (PRISMA) statement [18] (Supplementary file 1) and the International Society for Pharmacoeconomics and Outcomes Research Task Force on Indirect Treatment Comparisons Good Research Practices [19]. A formal protocol was not developed for this study.

\section{Study identification}

A systematic search of the databases PubMed, Cochrane Central Register of Controlled Trials, and Embase was conducted to capture all potential studies evaluating the prophylactic use of triazole, polyene, and echinocandin antifungal agents from their inception to April 2020. Each search strategy was modified depending on the specific requirements of the individual database under the assistance of a senior investigator. The reference lists of all eligible studies and topic-related reviews and the clinicaltrials.gov were also searched to include additional studies. The details of all search strategies for the three targeted databases after completing the electronic search are shown in Supplementary file 2. Any disagreement in study identification was resolved by consensus.

\section{Study selection}

Two investigators (Jie $\mathrm{Wu}$ and Jing Lai) were assigned to finish the study selection in the following three steps: (a) first, all duplicate records were eliminated using the $\mathrm{Du}$ plicates Elimination function of EndNote software; (b) the relevance of each record was evaluated by reviewing title and abstract; and (c) the eligibility of the remaining studies was checked by reviewing the full text eventually. Any divergence in study selection was resolved by consensus. Inclusion and exclusion criteria were developed to guide the study selection. The inclusion criteria were as follows: (a) adult patients with hematological malignancies receiving intensive chemotherapy for remission or HSCT; (b) randomized controlled trials (RCTs) comparing triazole, polyene, and echinocandin antifungal agents with placebo or with each other as prophylaxis against IFIs; (c) the overall incidence of proven or probable IFIs defined as the primary outcome, while the incidence of invasive Aspergillus and Candida infection, all-cause and IFI-related mortality, overall incidence of adverse events, withdrawal due to adverse events, need for empirical treatments, and proportion of successful treatment regarded as secondary outcomes; and (d) only studies published in English language.

A study was excluded if at least one of the following criteria was met: (a) studies without sufficient data and additional information not added through contacting the lead author and (b) duplicate study with relatively insufficient data.

\section{Data extraction}

Two investigators (Bing $\mathrm{Yu}$ and Bo Wang) independently extracted the following information, name of the first author, publication year, study design (multicenter and single center), country of the corresponding author, basic characteristics of participants (sample size, age, and sex ratio), details of treatments, follow-up time, outcomes, and details of the risk of bias. Any divergence in data extraction was resolved by consensus.

\section{Quality assessment}

The quality of eligible studies was assessed with the Cochrane risk-of-bias assessment tool [20] based on the random sequence generation; allocation concealment; blinding of participants and personnel; blinding of outcome assessment; incomplete outcome data; selective reporting; and other bias, which were performed by two independent investigators (Jie Wu and Jing Lai). A study 
was labeled as a low risk of bias if all items of the assessment tool were covered. A study was rated as a high risk of bias if at least one of the seven items was not fulfilled. Beyond that, a study was labeled as an unclear risk of bias. Any divergence in the quality assessment of studies was settled by consensus.

\section{Statistical analysis}

The data was statistically analyzed by two independent investigators (Zhuman $\mathrm{Wu}$ and Chengnian $\mathrm{Wu}$ ). In this systematic review and network meta-analysis, all outcomes of interest were dichotomous data. Therefore, the pooled risk ratio (RR) with $95 \%$ confidence intervals (CIs) was calculated to express it [21]. In a pairwise meta-analysis, heterogeneity across studies was first qualitatively assessed with the Cochrane $\mathrm{Q}$, and then $I^{2}$ statistic was used to quantitatively estimate the level of heterogeneity [22]. Studies were deemed to be homogeneous if $P>0.1$ and $I^{2}<50$. Otherwise, studies were considered as heterogeneous when $P<0.1$ and $I^{2}>50$. All traditional head-to-head meta-analyses were performed with the random-effects model, which simultaneously considered within- and between-study heterogeneity. Publication bias was checked by drawing a funnel plot when the number of eligible studies for individual outcome was more than 10 [23], and an asymmetry suggested publication bias [24]. Traditional pairwise metaanalysis was performed using Review Manager 5.3 (Cochrane Collaboration, Copenhagen, Denmark).

Random-effects network meta-analyses were conducted using Markov Chain Monte-Carlo Methods in OpenBUGS 3.2.3 (MRC Biostatistics Unit, Cambridge, UK) following the methods described by $\mathrm{Lu}$ and Ades $[25,26]$. The initial value automatically generated from the software was used to fit the model [27]. The Markov Chain Monte-Carlo method with 50,000 iterations and 20,000 burn-in was used to gain convergence. The summary treatment effect estimates were presented as odds ratios (ORs), with 95\% credible interval (CrI) for treatment comparisons. The comparison-adjusted funnel plot was drawn to assess the small-study effects when the number of studies included in one pair of comparison was more than 10 [28]. The inconsistency factor was calculated using the loop-specific method to assess the inconsistency [29]. The ranking probabilities of being at each possible rank were estimated for all treatments, and the surface under the cumulative ranking curve values was used to provide a hierarchy of treatments [30].

\section{Results}

\section{Study selection}

The flow diagram of study retrieval and selection is shown in Fig. 1. A total of 239 records were captured after initially searching 3 targeted databases. After removing duplicate records, checking the eligibility of the remaining studies, and then adding additional eligible studies, 35 studies [31-44] involving 37 RCTs were included in this network meta-analysis. The reasons for excluding ineligible studies according to the selection criteria are summarized in Fig. 1.

\section{Study characteristics}

The characteristics of all eligible studies are listed in Table 1. Moreover, the details of outcomes of interest are summarized in Table 2. The studies were reported between 1993 and 2019. Of these 35 studies, 16 [1, 2, 38, 40, 43-50] used multiple-center design, 14 [2, 13, 33, 37, $38,41,42,44,46,47,49,51]$ did not report details of follow-up, 1 [45] was a three-arm design, and $2[49,52]$ were retrieved from clinicaltrial.gov. The sample size of individual study varied from 25 to 602, with 8513 participants. In total, seven active drugs and placebo were identified. Further, 17 comparisons were identified, and fluconazole was found to be the most extensively studied. The associations among the seven active antifungals and placebo are delineated in Fig. 2.

\section{Quality assessment}

The risk of bias of all eligible studies is depicted in Fig. 3. Overall, most of the studies (60.0\%) [2, 13, 15, 32, 34, 41, $42,44,48,51-54]$ had a high risk of bias, and only four studies $[1,40,45,46]$ had a low risk of bias. Of these 35 studies, 19 (54.3\%) [12, 14, 15, 31-34, 37, 38, 42-44, 49, $50,52-55]$ did not describe the methodology of generating random sequence, $21(60.0 \%)[12-15,31-34,37,38$, $42,47,49-54,56-58]$ did not report the details for allocation concealment, and $2(5.7 \%)$ [41, 44] did not conceal random sequence. Four studies (11.4\%) [14, 41, 44, 48] used open-label design, and seven (20.0\%) [1, 31, 40, $45,46,49,58]$ reported the details of blinding personnel, participants, and outcome assessors. Attrition bias was detected among eight studies (22.9\%) [13, 31, 32, 35, 42, 53, 55, 57] because appropriate methods of addressing incomplete data were not implemented. All studies reported the anticipated outcomes as specified in the Methods section. Other bias sources were not detected in all studies.

\section{Direct treatment effects Primary outcome}

According to the associations among all targeted drugs, direct meta-analyses were performed on proven and probable IFIs, which are delineated in Supplementary file 3 (Fig. S1). Pooled results suggested that fluconazole cloud reduced the incidence of proven and probable IFIs compared with placebo (7 trials; RR, 0.41; 95\% CI, 0.25 $\left.0.69 ; P<0.001 ; I^{2}=37 \%\right)$. However, the effect of fluconazole in reducing proven and probable IFIs was inferior 


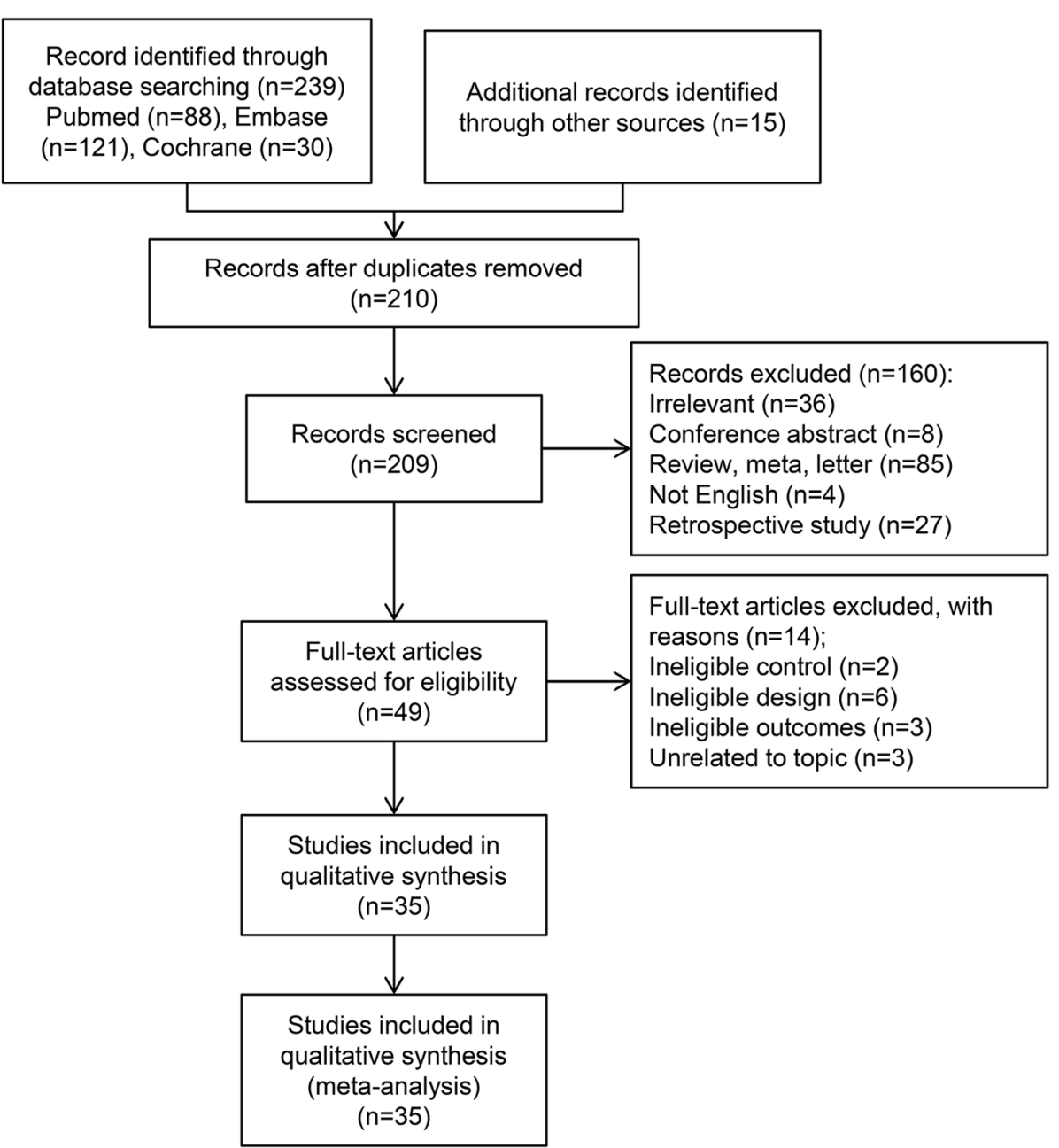

Fig. 1 Flow diagram of study retrieval and selection. CENTRAL, Cochrane Central Register of Controlled Trials

to that of posaconazole ( 2 trials; RR, 3.17; 95\% CI, $1.61-$ 6.23; $P=0.008 ; I^{2}=0 \%$ ) and caspofungin (1 trial; RR, $2.81 ; 95 \%$ CI, $1.13-7.01 ; P=0.03 ; I^{2}=$ n.a. $)$. No significant difference was detected among the remaining comparisons.

\section{Secondary outcomes}

All comparisons investigating IA-related IFIs are delineated in Supplementary file 3 (Fig. S2). The metaanalysis showed a beneficial result for posaconazole compared with fluconazole (1 trial; RR, 9.50; 95\% CI, $2.19-41.14 ; P=0.003 ; I^{2}=$ n.a. $)$ and itraconazole ( 1 trial; RR, 13.57; 95\% CI, 2.70-68.23; $P=0.002 ; I^{2}=$ n.a.), but no significant pooled result was detected for other comparisons. Two comparisons reported the incidence of invasive Candida (IC)-related IFIs, and meta-analyses suggested that fluconazole (5 trials; RR, 0.34; 95\% CI, $0.14-0.85 ; P=0.02 ; I^{2}=43 \%$ ) and itraconazole (2 trials; RR, $0.25 ; 95 \%$ CI, $\left.0.07-0.88 ; P=0.03 ; I^{2}=0 \%\right)$ reduced the incidence of IC-related IFIs. All pooled results are displayed in Supplementary file 3 (Fig. S3).

Thirteen comparisons reported the incidence of allcause mortality, and the meta-analysis did not identify significant differences. All pooled results are delineated in Supplementary file 3 (Fig. S4). Moreover, nine comparisons also reported the incidence of IFI-related mortality. No significant difference was observed among all comparisons, which are delineated in Supplementary file 3 (Fig. S5).

Thirteen comparisons reported the incidence of adverse events. The meta-analysis suggested that fluconazole (5 trials; RR, $0.64 ; 95 \% \mathrm{CI}, 0.42-0.96 ; P=0.03 ; I^{2}=$ $78 \%)$ was associated with the reduced incidence of adverse events compared with itraconazole, and micafungin was associated with a reduced incidence of adverse events compared with posaconazole ( 1 trial; RR, 17.93; 95\% CI, 2.47-130.20; $P=0.004 ; I^{2}=$ n.a.). No significant difference was detected among the remaining pooled 


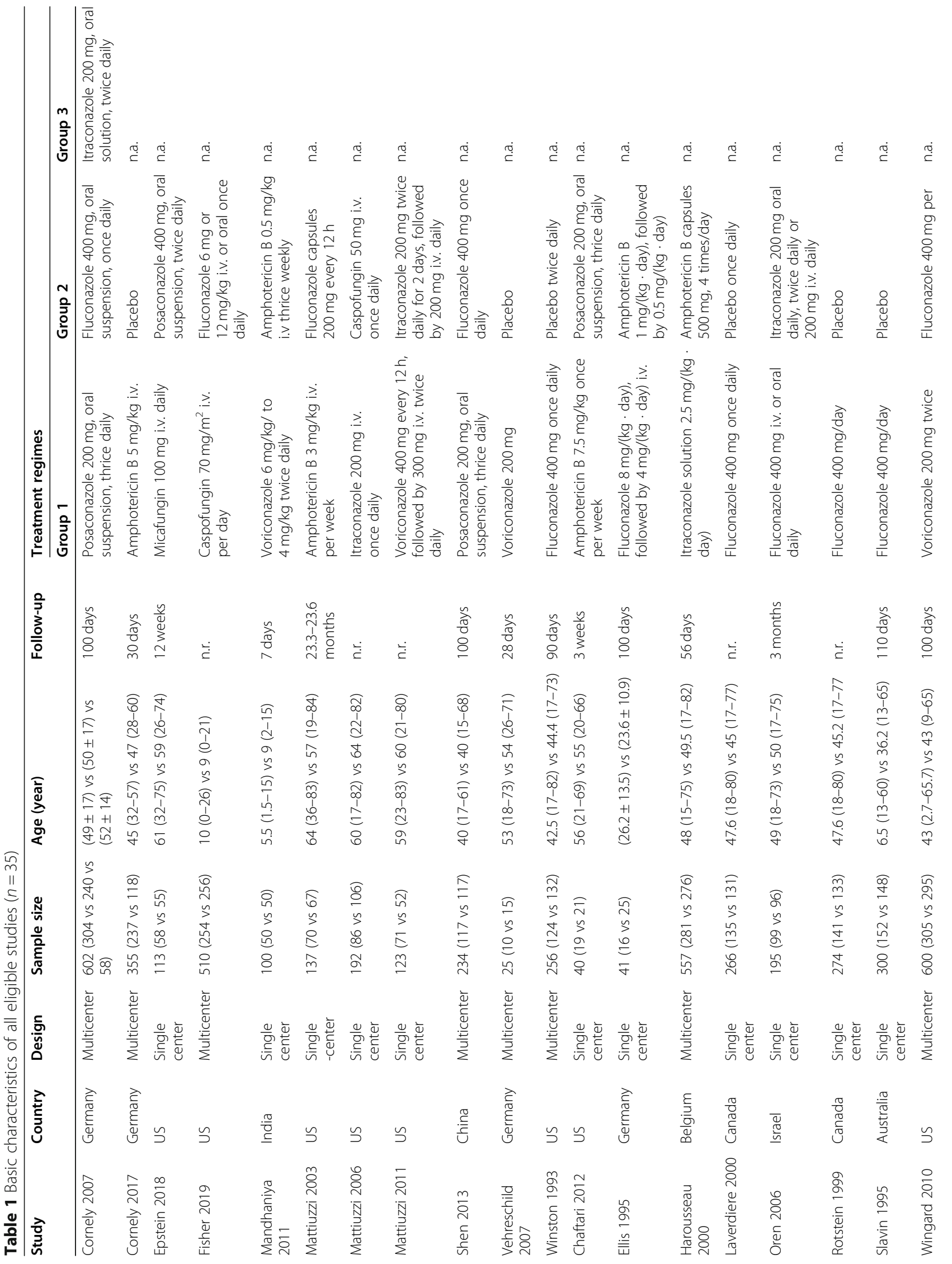


ํํำ

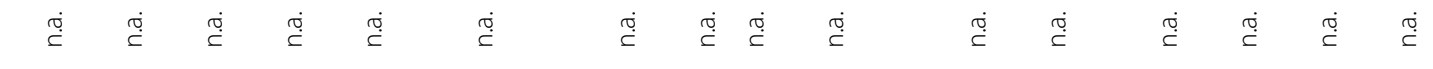

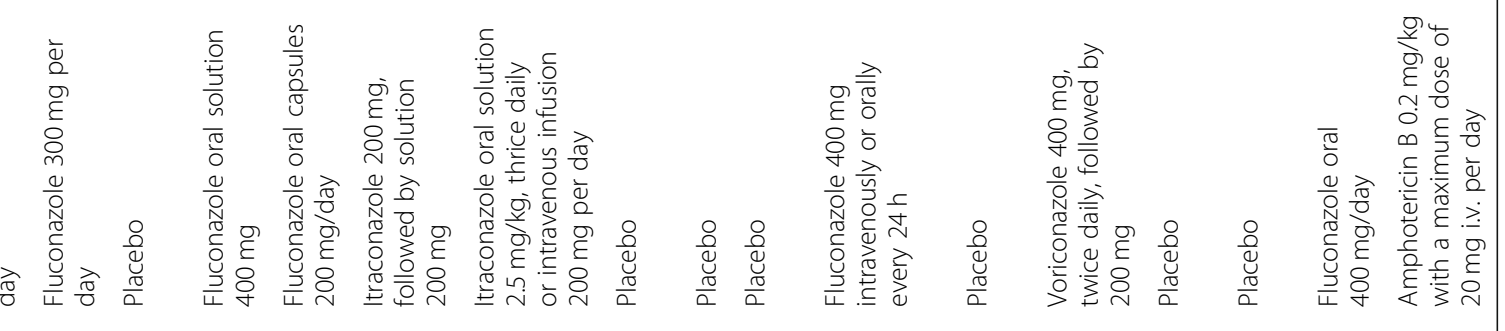

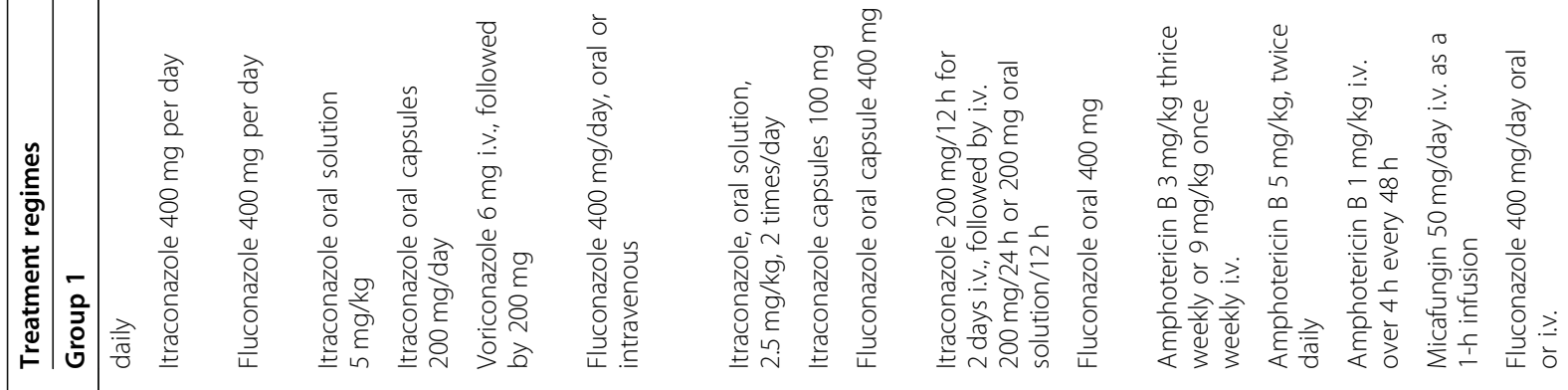
욱
3̀
은



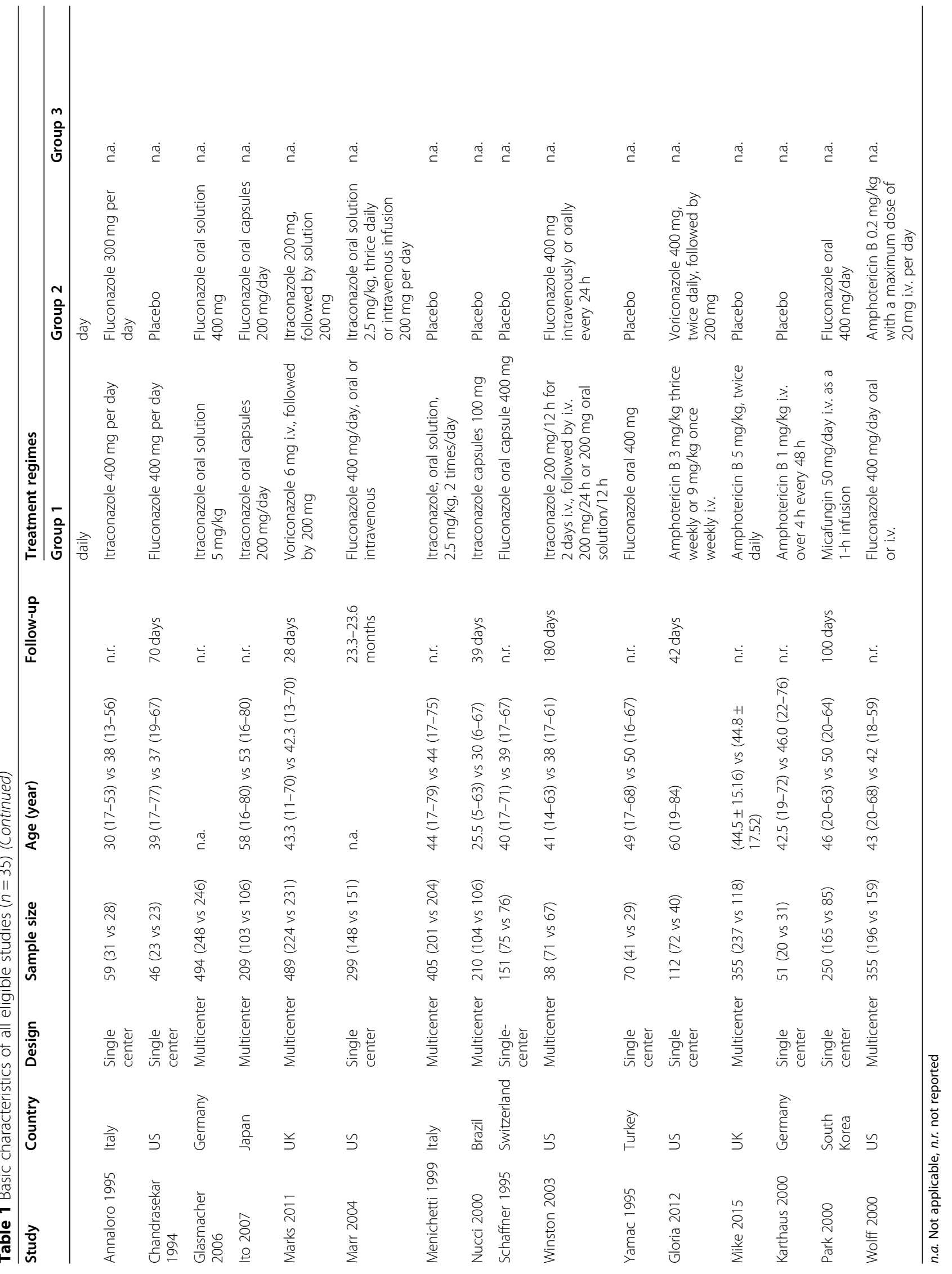


Table 2 Outcomes of 35 eligible studies

\begin{tabular}{|c|c|c|c|c|c|c|c|c|c|c|c|}
\hline \multirow[t]{2}{*}{ Study } & \multirow[t]{2}{*}{ Regimes } & \multirow{2}{*}{$\begin{array}{l}\text { Sample } \\
\text { size }\end{array}$} & \multicolumn{3}{|l|}{ IFIs } & \multicolumn{2}{|c|}{ Mortality } & \multicolumn{2}{|l|}{ AEs } & \multirow{2}{*}{$\begin{array}{l}\text { Empirical } \\
\text { treatment }\end{array}$} & \multirow{2}{*}{$\begin{array}{l}\text { Successful } \\
\text { treatment }\end{array}$} \\
\hline & & & $\begin{array}{l}\text { Probable/ } \\
\text { Proven }\end{array}$ & IA & IC & $\begin{array}{l}\text { All- } \\
\text { cause }\end{array}$ & $\begin{array}{l}\text { IFI- } \\
\text { related }\end{array}$ & All & $\begin{array}{l}\text { Withdrawal } \\
\text { due to AEs }\end{array}$ & & \\
\hline \multirow[t]{3}{*}{ Cornely 2007} & Posaconazole & 304 & 7 & 2 & 3 & n.r. & n.r. & 159 & 19 & n.r. & n.r. \\
\hline & Fluconazole & 240 & 19 & 15 & 2 & n.r. & n.r. & 143 & 4 & n.r. & n.r. \\
\hline & Itraconazole & 58 & 6 & 5 & 0 & n.r. & n.r. & 32 & 2 & n.r. & n.r. \\
\hline \multirow[t]{2}{*}{ Cornely 2017} & Amphotericin B & 237 & 18 & n.r. & 1 & 17 & 2 & 237 & 226 & 37 & 142 \\
\hline & Placebo & 118 & 13 & n.r. & 3 & 8 & 0 & 115 & 110 & 24 & 77 \\
\hline \multirow[t]{2}{*}{ Epstein 2018} & Micafungin & 58 & 5 & 2 & 0 & 7 & 2 & 1 & n.r. & n.r. & 38 \\
\hline & Posaconazole & 55 & 3 & 0 & 1 & 2 & 0 & 17 & n.r. & n.r. & 26 \\
\hline \multirow[t]{2}{*}{ Fisher 2019} & Caspofungin & 254 & 6 & 2 & n.r. & n.r. & n.r. & 83 & n.r. & 160 & n.r. \\
\hline & Fluconazole & 256 & 17 & 5 & n.r. & n.r. & n.r. & 98 & n.r. & 162 & n.r. \\
\hline \multirow[t]{2}{*}{ Mandhaniya 2011} & Voriconazole & 50 & 1 & n.r. & n.r. & 1 & n.r. & 22 & 3 & 11 & 36 \\
\hline & Amphotericin B & 50 & 0 & n.r. & n.r. & 2 & n.r. & 16 & 15 & 13 & 33 \\
\hline \multirow[t]{2}{*}{ Mattiuzzi 2003} & Amphotericin B & 70 & 3 & n.r. & n.r. & 10 & 1 & 10 & n.r. & n.r. & 34 \\
\hline & Fluconazole & 67 & 3 & n.r. & n.r. & 8 & 1 & 5 & n.r. & n.r. & 32 \\
\hline \multirow[t]{2}{*}{ Mattiuzzi 2006} & Itraconazole & 86 & 5 & 1 & 4 & 7 & 2 & n.r. & 8 & n.r. & 44 \\
\hline & Caspofungin & 106 & 7 & 2 & 2 & 7 & 4 & n.r. & 4 & n.r. & 55 \\
\hline \multirow[t]{2}{*}{ Mattiuzzi 2011} & Voriconazole & 71 & 0 & 0 & n.r. & 6 & n.r. & 15 & n.r. & 21 & 48 \\
\hline & Itraconazole & 52 & 2 & 1 & n.r. & 6 & n.r. & 6 & n.r. & 20 & 29 \\
\hline \multirow[t]{2}{*}{ Shen 2013} & Posaconazole & 117 & 4 & n.r. & n.r. & 3 & n.r. & 25 & n.r. & 11 & 80 \\
\hline & Fluconazole & 117 & 11 & n.r. & n.r. & 7 & n.r. & 15 & n.r. & 27 & 68 \\
\hline \multirow[t]{2}{*}{ Vehreschild 2007} & Voriconazole & 10 & n.r. & n.r. & n.r. & 0 & n.r. & 3 & n.r. & n.r. & n.r. \\
\hline & Placebo & 15 & n.r. & n.r. & n.r. & 2 & n.r. & 6 & n.r. & n.r. & n.r. \\
\hline \multirow[t]{2}{*}{ Winston 1993} & Fluconazole & 124 & 5 & 3 & 2 & 26 & n.r. & n.r. & n.r. & n.r. & n.r. \\
\hline & Placebo & 132 & 10 & 3 & 7 & 24 & n.r. & n.r. & n.r. & n.r. & n.r. \\
\hline \multirow[t]{2}{*}{ Chaftari 2012} & Amphotericin B & 19 & 1 & n.r. & n.r. & n.r. & n.r. & 19 & n.r. & n.r. & n.r. \\
\hline & Posaconazole & 21 & 0 & n.r. & n.r. & n.r. & n.r. & 20 & n.r. & n.r. & n.r. \\
\hline \multirow[t]{2}{*}{ Ellis 1995} & Fluconazole & 16 & 6 & 4 & 2 & 8 & 5 & 0 & 0 & n.r. & n.r. \\
\hline & Amphotericin B & 25 & 3 & 2 & 0 & 6 & 2 & 20 & 3 & n.r. & n.r. \\
\hline \multirow[t]{2}{*}{ Harousseau 2000} & Itraconazole & 281 & 8 & 5 & 2 & 18 & 1 & 222 & 13 & 114 & 206 \\
\hline & Amphotericin B & 276 & 14 & 9 & 3 & 23 & 5 & 205 & 13 & 132 & 198 \\
\hline Laverdiere 2000 & Fluconazole & 135 & 9 & 1 & 8 & n.r. & n.r. & n.r. & n.r. & n.r. & n.r. \\
\hline & Placebo & 131 & 32 & 8 & 23 & n.r. & n.r. & n.r. & n.r. & n.r. & n.r. \\
\hline Oren 2006 & Fluconazole & 99 & 12 & 11 & 1 & 11 & 8 & n.r. & n.r. & n.r. & n.r. \\
\hline & Itraconazole & 96 & 11 & 9 & 2 & 9 & 3 & n.r. & n.r. & n.r. & n.r. \\
\hline Rotstein 1999 & Fluconazole & 141 & 9 & n.r. & 3 & 15 & 1 & n.r. & n.r. & n.r. & 81 \\
\hline & Placebo & 133 & 32 & n.r. & 20 & 15 & 6 & n.r. & n.r. & n.r. & 67 \\
\hline Slavin 1995 & Fluconazole & 152 & 10 & n.r. & n.r. & 31 & 6 & n.r. & 57 & n.r. & n.r. \\
\hline & Placebo & 148 & 16 & n.r. & n.r. & 52 & 13 & n.r. & 81 & n.r. & n.r. \\
\hline Wingard 2010 & Voriconazole & 305 & 22 & 9 & 3 & n.r. & n.r. & 21 & n.r. & 73 & n.r. \\
\hline & Fluconazole & 295 & 23 & 17 & 3 & n.r. & n.r. & 18 & n.r. & 89 & n.r. \\
\hline Annaloro 1995 & Itraconazole & 31 & 4 & n.r. & n.r. & 2 & n.r. & n.r. & n.r. & 16 & n.r. \\
\hline & Fluconazole & 28 & 1 & n.r. & n.r. & 2 & n.r. & n.r. & n.r. & 12 & n.r. \\
\hline
\end{tabular}


Table 2 Outcomes of 35 eligible studies (Continued)

\begin{tabular}{|c|c|c|c|c|c|c|c|c|c|c|c|}
\hline \multirow[t]{2}{*}{ Study } & \multirow[t]{2}{*}{ Regimes } & \multirow{2}{*}{$\begin{array}{l}\text { Sample } \\
\text { size }\end{array}$} & \multicolumn{3}{|l|}{ IFIs } & \multicolumn{2}{|c|}{ Mortality } & \multicolumn{2}{|l|}{ AEs } & \multirow{2}{*}{$\begin{array}{l}\text { Empirical } \\
\text { treatment }\end{array}$} & \multirow{2}{*}{$\begin{array}{l}\text { Successfu } \\
\text { treatment }\end{array}$} \\
\hline & & & $\begin{array}{l}\text { Probable/ } \\
\text { Proven }\end{array}$ & IA & $\overline{I C}$ & $\begin{array}{l}\text { All- } \\
\text { cause }\end{array}$ & $\begin{array}{l}\text { IFI- } \\
\text { related }\end{array}$ & $\overline{\text { All }}$ & $\begin{array}{l}\text { Withdrawal } \\
\text { due to AEs }\end{array}$ & & \\
\hline \multirow[t]{2}{*}{ Chandrasekar 1994} & Fluconazole & 23 & 2 & 2 & 0 & 4 & 2 & n.r. & n.r. & n.r. & 5 \\
\hline & Placebo & 23 & 1 & 0 & 1 & 2 & 1 & n.r. & n.r. & n.r. & 14 \\
\hline \multirow[t]{2}{*}{ Glasmacher 2006} & Itraconazole & 248 & 4 & 2 & 1 & 25 & 2 & 90 & 15 & n.r. & n.r. \\
\hline & Fluconazole & 246 & 5 & 3 & 1 & 28 & 3 & 61 & 12 & n.r. & n.r. \\
\hline \multirow[t]{2}{*}{ Ito 2007} & Itraconazole & 103 & 1 & n.r. & n.r. & n.r. & n.r. & 4 & n.r. & 21 & n.r. \\
\hline & Fluconazole & 106 & 3 & n.r. & n.r. & n.r. & n.r. & 2 & n.r. & 20 & n.r. \\
\hline \multirow[t]{2}{*}{ Marks 2011} & Voriconazole & 224 & 3 & 1 & 2 & 59 & n.r. & n.r. & n.r. & 67 & 109 \\
\hline & Itraconazole & 231 & 5 & 5 & 0 & 80 & n.r. & n.r. & n.r. & 101 & 80 \\
\hline \multirow[t]{2}{*}{ Marr 2004} & Fluconazole & 148 & 22 & 7 & 4 & 44 & 11 & 23 & n.r. & 25 & n.r. \\
\hline & Itraconazole & 151 & 11 & 8 & 3 & 55 & 12 & 52 & n.r. & 19 & n.r. \\
\hline \multirow[t]{2}{*}{ Menichetti 1999} & Itraconazole & 201 & 5 & 4 & 1 & 15 & 1 & n.r. & 37 & 43 & 166 \\
\hline & Placebo & 204 & 9 & 1 & 7 & 18 & 5 & n.r. & 27 & 59 & 146 \\
\hline \multirow[t]{2}{*}{ Nucci 2000} & Itraconazole & 104 & 5 & 1 & 2 & 8 & 2 & n.r. & 6 & 26 & 76 \\
\hline & Placebo & 106 & 9 & 1 & 6 & 7 & 1 & n.r. & 7 & 36 & 63 \\
\hline \multirow[t]{2}{*}{ Schaffner 1995} & Fluconazole & 75 & 8 & 4 & 4 & 5 & 2 & n.r. & n.r. & 36 & n.r. \\
\hline & Placebo & 76 & 8 & 7 & 0 & 4 & 2 & n.r. & n.r. & 25 & n.r. \\
\hline \multirow[t]{2}{*}{ Winston 2003} & Itraconazole & 71 & 6 & 3 & 2 & 32 & 6 & 33 & n.r. & n.r. & n.r. \\
\hline & Fluconazole & 67 & 17 & 8 & 8 & 28 & 12 & 14 & n.r. & n.r. & n.r. \\
\hline \multirow[t]{2}{*}{ Yamac 1995} & Fluconazole & 41 & 4 & n.r. & n.r. & n.r. & n.r. & n.r. & n.r. & n.r. & n.r. \\
\hline & Placebo & 29 & 8 & n.r. & n.r. & n.r. & n.r. & n.r. & n.r. & n.r. & n.r. \\
\hline \multirow[t]{2}{*}{ Gloria 2012} & Amphotericin B & 72 & 7 & n.r. & n.r. & 2 & n.r. & 7 & n.r. & n.r. & n.r. \\
\hline & Voriconazole & 40 & 2 & n.r. & n.r. & 2 & n.r. & 4 & n.r. & n.r. & n.r. \\
\hline \multirow[t]{2}{*}{ Mike 2015} & Amphotericin B & 237 & 18 & n.r. & n.r. & n.r. & n.r. & n.r. & n.r. & n.r. & 142 \\
\hline & Placebo & 118 & 12 & n.r. & n.r. & n.r. & n.r. & n.r. & n.r. & n.r. & 77 \\
\hline \multirow[t]{2}{*}{ Karthaus 2000} & Amphotericin B & 20 & 2 & n.r. & n.r. & 5 & 0 & n.r. & n.r. & 9 & n.r. \\
\hline & Placebo & 31 & 6 & n.r. & n.r. & 9 & 3 & n.r. & n.r. & 29 & n.r. \\
\hline \multirow[t]{2}{*}{ Park 2000} & Micafungin & 165 & 12 & 1 & 1 & 15 & 2 & n.r. & n.r. & n.r. & 155 \\
\hline & Fluconazole & 85 & 7 & 0 & 1 & 11 & 3 & n.r. & n.r. & n.r. & 77 \\
\hline \multirow[t]{2}{*}{ Wolff 2000} & Fluconazole & 196 & 8 & 2 & 8 & 24 & n.r. & n.r. & n.r. & n.r. & 103 \\
\hline & Amphotericin B & 159 & 12 & 1 & 11 & 19 & n.r. & n.r. & n.r. & n.r. & 67 \\
\hline
\end{tabular}

AEs Adverse events, IA invasive Aspergillus infections, IC invasive Candida, IFls invasive fungal infections, n.r. not reported

comparisons. All pooled results are delineated in Supplementary file 3 (Fig. S6). Moreover, 10 comparisons reported withdrawal due to adverse events. The metaanalysis suggested that the incidence of withdrawal due to adverse events in patients receiving fluconazole was lower than that in patients receiving placebo ( 1 trial; RR, 0.69 ; 95\% CI, $0.53-0.88 ; P=0.003 ; I^{2}=$ n.a.) and posaconazole ( 1 trial; RR, 0.27 ; 95\% CI, $0.09-0.77 ; P=0.01 ; I^{2}=$ n.a.). The meta-analysis also indicated a beneficial result for voriconazole ( 1 trial; RR, 0.20 ; $95 \% \mathrm{CI}, 0.06-0.65$; $P=0.007 ; I^{2}=$ n.a.) compared with amphotericin B for withdrawal due to adverse events. All pooled results are delineated in Supplementary file 3 (Fig. S7).
Ten comparisons reported the need for empirical treatment. Significant differences were detected when fluconazole was related to posaconazole ( 1 trial; RR, 2.45 ; $95 \% \mathrm{CI}$, $1.28-4.71 ; P=0.007 ; I^{2}=$ n.a.), itraconazole was related to voriconazole (2 trials; RR, 1.43 ; $95 \% \mathrm{CI}, 1.14-1.78 ; P=$ $0.002 ; I^{2}=0 \%$ ) or placebo ( 2 trials; RR, $0.74 ; 95 \%$ CI, $0.57-$ $0.96 ; P=0.03 ; I^{2}=0 \%$ ), and amphotericin B was related to placebo ( 2 trials; RR, $0.61 ; 95 \% \mathrm{CI}, 0.38-0.98 ; P=0.04$; $\left.I^{2}=48 \%\right)$. No significant difference was detected among the remaining comparisons. All pooled results are delineated in Supplementary file 3 (Fig. S8).

Eleven comparisons reported the proportion of successful treatment. The meta-analysis suggested that 


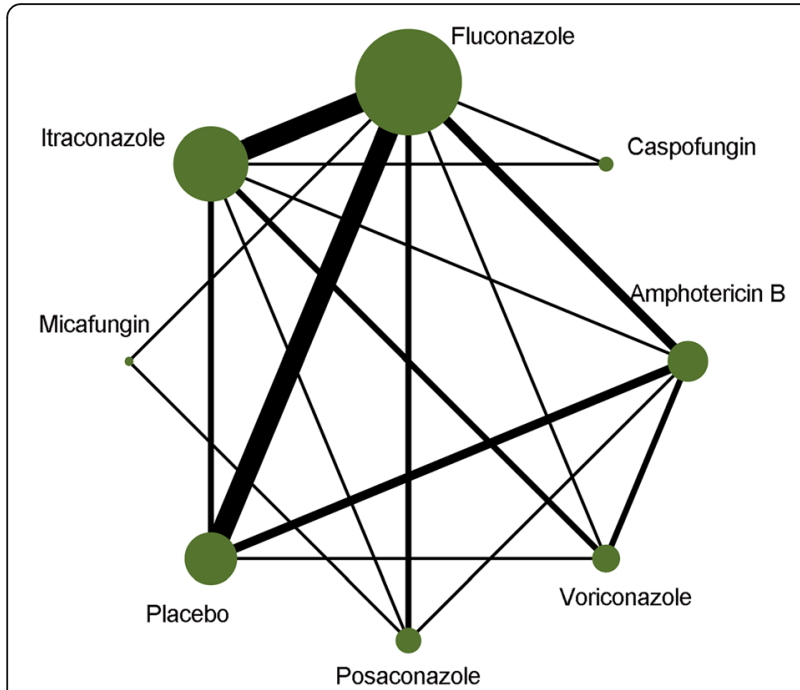

Fig. 2 Network of all direct comparisons between antifungal agents and placebo. The sizes of the nodes indicate the numbers of participants, and the widths of the lines indicate the numbers of included trials

fluconazole was associated with an increased proportion of successful treatment compared with amphotericin B ( 2 trials; RR, 1.24; 95\% CI, 1.02-1.50; $P=0.03 ; I^{2}=0 \%$ ). Moreover, the meta-analysis also suggested that itraconazole was associated with the increased proportion of successful treatment compared with placebo ( 2 trials; RR, 1.17; 95\% CI, 1.07-1.29; $P=0.001 ; I^{2}=0 \%$ ); however, itraconazole was inferior to voriconazole ( 2 trials; RR, $0.75 ; 95 \% \mathrm{CI}, 0.63-0.90 ; P=0.002 ; I^{2}=0 \%$ ). All pooled results are delineated in Supplementary file 3 (Fig. S9).

\section{Network meta-analysis}

\section{Primary outcome}

The network meta-analysis was performed to calculate mixed effect estimates. Compared with placebo, fluconazole (OR, 2.19; 95\% CrI, 1.39-3.16), itraconazole (OR, 2.92; 95\% CrI, 1.64-4.63), posaconazole (OR, 8.51; 95\% CrI, 3.25-18.72), voriconazole (OR, 3.40; 95\% CrI, 1.417.14), amphotericin B (OR, 1.80; 95\% CrI, 1.04-2.95), caspofungin (OR, 4.85; 95\% CrI, 1.54-11.27), but not micafungin (OR, 3.46; 95\% CrI, 0.95-9.06), reduced the incidence of proven and probable IFIs (Table 3). Moreover, the network meta-analysis also suggested that posaconazole was superior to fluconazole (OR, 0.30; 95\% CrI, 0.12-0.60), itraconazole (OR, 0.40; 95\% CrI, 0.15-0.85), and amphotericin B (OR, 4.97; 95\% CrI, 1.73-11.35) in reducing the incidence of proven and probable IFIs.

The hierarchies of all drugs were generated on the basis of SUCRA values for prophylaxis against proven and probable IFIs. The results indicated that

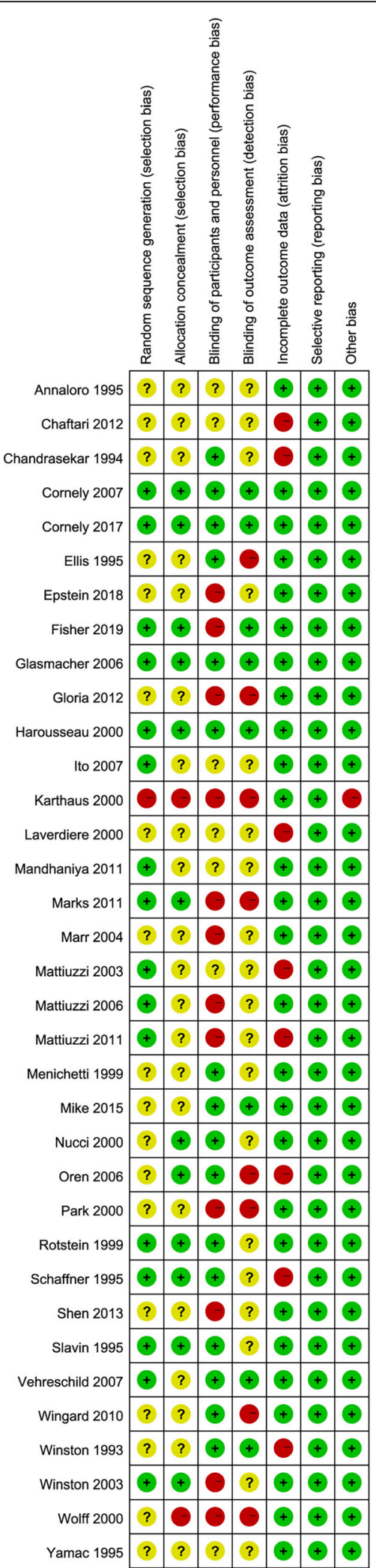

Fig. 3 Risk-of-bias summary. The red (-), yellow (?), and green (+) represent high, unclear, and low risk of bias, respectively 
Table 3 Pooled summary estimates derived from direct and network meta-analyses on the comparative efficacy of prophylaxis antifungal agents against IFIs

\begin{tabular}{|c|c|c|}
\hline Comparisons & Direct estimate, OR $(95 \% \mathrm{Cl})$ & Network meta-analysis, OR $(95 \% \mathrm{Crl})$ \\
\hline \multicolumn{3}{|c|}{ Compared with fluconazole } \\
\hline Itraconazole & $0.71(0.44-1.15)$ & $0.78(0.50-1.15)$ \\
\hline Posaconazole & $0.32(0.16-0.62)$ & $0.30(0.12-0.60)$ \\
\hline Voriconazole & $0.93(0.53-1.62)$ & $0.73(0.31-1.38)$ \\
\hline Amphotericin B & $0.96(0.33-2.83)$ & $1.28(0.71-2.04)$ \\
\hline Caspofungin & $0.36(0.14-0.89)$ & $0.56(0.20-1.27)$ \\
\hline Micafungin & $0.88(0.36-2.16)$ & $0.84(0.25-2.11)$ \\
\hline Placebo & $2.20(1.42-3.42)$ & $2.19(1.39-3.16)$ \\
\hline \multicolumn{3}{|c|}{ Compared with itraconazole } \\
\hline Posaconazole & $0.21(0.08-0.62)$ & $0.40(0.15-0.85)$ \\
\hline Voriconazole & $0.48(0.13-1.72)$ & $0.98(0.40-1.92)$ \\
\hline Amphotericin B & $1.78(0.76-4.18)$ & $1.70(0.86-2.85)$ \\
\hline Caspofungin & $1.14(0.37-3.45)$ & $0.74(0.26-1.68)$ \\
\hline Micafungin & - & $1.13(0.31-2.92)$ \\
\hline Placebo & $1.77(0.83-3.76)$ & $2.92(1.64-4.63)$ \\
\hline \multicolumn{3}{|c|}{ Compared with posaconazole } \\
\hline Voriconazole & - & $2.85(0.83-7.08)$ \\
\hline Amphotericin B & $3.30(0.14-76.46))$ & $4.97(1.73-11.35)$ \\
\hline Caspofungin & - & $2.20(0.55-6.24)$ \\
\hline Micafungin & $1.58(0.40-6.30)$ & $3.13(0.85-8.32)$ \\
\hline Placebo & - & $8.51(3.25-18.72)$ \\
\hline \multicolumn{3}{|c|}{ Compared with voriconazole } \\
\hline Amphotericin B & $1.40(0.35-5.52)$ & $1.96(0.80-4.06)$ \\
\hline Caspofungin & - & $0.87(0.23-2.41)$ \\
\hline Micafungin & - & $1.32(0.30-4.01)$ \\
\hline Placebo & - & $3.40(1.41-7.14)$ \\
\hline \multicolumn{3}{|c|}{ Compared with amphotericin B } \\
\hline Caspofungin & - & $0.47(0.14-1.20)$ \\
\hline Micafungin & - & $0.71(0.19-1.95)$ \\
\hline Placebo & $1.11(0.66-1.87)$ & $1.80(1.04-2.95)$ \\
\hline \multicolumn{3}{|c|}{ Compared with caspofungin } \\
\hline Micafungin & - & $1.88(0.35-5.81)$ \\
\hline Placebo & - & $4.85(1.54-11.27)$ \\
\hline \multicolumn{3}{|c|}{ Compared with micafungin } \\
\hline Placebo & - & $3.46(0.95-9.06)$ \\
\hline
\end{tabular}

Numbers in bold are statistically significant differences

$\mathrm{Cl}$ Confidence interval, $\mathrm{Cr}$ credible interval, IFls invasive fungal infections, OR odds ratio

posaconazole had the highest probability of being ranked the best $(99.2 \%)$, followed by voriconazole $(77.9 \%)$, itraconazole (66.0\%), fluconazole (45.1\%), caspofungin (44.0\%), micafungin (38.5\%), and amphotericin B (24.5\%). The plot of rankings of all treatments is delineated in Supplementary file 3 (Fig. S10).

\section{Secondary outcomes}

The network meta-analysis showed that fluconazole (OR, 0.08; 95\% CrI, 0.01-0.27), itraconazole (OR, 0.13; 95\% CrI, 0.01-0.44), voriconazole (OR, 15.07; 95\% CrI, 1.09-76.67), amphotericin B (OR, 38.32; 95\% CrI, 2.97184.9), micafungin (OR, 41.39; 95\% CrI, $2.43-212.8)$, and 
placebo (OR, 4.78; 95\% CrI, 4.08 to 218.0 ), but not caspofungin (OR, 24.43; 95\% CrI, 0.98-139.1), all increased the incidence of IA-related IFIs compared with posaconazole (Table 4). Itraconazole also reduced the incidence of IC-related IFI compared with placebo (OR, 8.27; 95\% CrI, 1.51-26.57) (Table 4).

The network meta-analysis demonstrated that fluconazole (OR, 0.35; 95\% CrI, 0.08-0.96), itraconazole (OR, 0.33; 95\% CrI, 0.07-0.94), amphotericin B (OR, 4.49; 95\% CrI, 1.04-13.86), and placebo (OR, 4.98; 95\% CrI, 1.16-15.28), but not voriconazole (OR, 3.34; 95\% CrI, 0.67-10.58), caspofungin (OR, 4.34; 95\% CrI, 0.5416.84), and micafungin (OR, 3.23; 95\% CrI, 0.85-9.95), increased all-cause mortality compared with posaconazole (Table 4). Itraconazole also reduced the incidence of IFI-related mortality compared with placebo (OR, 3.39; 95\% CrI, 1.07-8.30) (Table 4).

The network meta-analysis showed that fluconazole (OR, 0.02; 95\% CrI, 0.00-0.03), itraconazole (OR, 0.01; 95\% CrI, 0.00-0.02), posaconazole (OR, 0.02; 95\% CrI, 0.00-0.03), voriconazole (OR, 0.005; 95\% CrI, 0.000.01), amphotericin B (OR, 0.004; 95\% CrI, 0.00-0.01), and caspofungin (OR, 0.05; 95\% CrI, 0.00-0.42), but not micafungin (OR, 1.39; 95\% CrI, 0.00-1.52), were associated with a reduced incidence of adverse events compared with placebo (Table 4). Fluconazole (OR, 0.12; 95\% CrI, 0.00-0.61), itraconazole (OR, 0.06; 95\% CrI, 0.00-0.29), posaconazole (OR, 0.07; 95\% CrI, 0.00-0.37), voriconazole (OR, 0.05; 95\% CrI, 0.00-0.23), and amphotericin B (OR, 0.04; 95\% CrI, 0.00-0.19), but not caspofungin (OR, 0.66; 95\% CrI, 0.00-1.35), reduced the incidence of adverse events compared with micafungin. Moreover, fluconazole was associated with an increased incidence of adverse events compared with amphotericin B (OR, 4.15; 95\% CrI, 1.20-12.08). The network metaanalysis also demonstrated that fluconazole and voriconazole were superior to posaconazole (OR, 6.58; $95 \%$ CrI, 1.07-18.26) and amphotericin B (OR, 14.84; 95\% CrI, 1.40-48.77), respectively (Table 4).

The network meta-analysis showed no significant difference among all comparisons in terms of the need for empirical treatment and the proportion of successful treatment (Table 4).

\section{Publication bias and network coherence}

The split-node method was adopted to generate the inconsistency plot so as to check the consistency of results from direct and indirect comparisons. The results of inconsistency plot indicated consistency in terms of proven and probable IFIs (Fig. 4). No evidence of publication bias based on comparison-adjusted funnel plot asymmetry was found (Fig. 5), although the number of studies included in each comparison was very small, thereby making the available methods for evaluating publication bias somewhat unreliable.

\section{Discussion}

IFIs remain a leading cause of morbidity and mortality among patients at high risk $[4,7]$ due to elusive identification of IFIs in the early stage $[8,9]$. Therefore, prophylaxis strategies are crucial in the containment of IFIs [3]. Previous traditional direct meta-analyses and network meta-analyses did not consider all prophylaxis treatments and did not incorporate all potentially eligible studies, thus restricting the reference value of previous findings for making decisions in clinical practice. The present network meta-analysis was performed on 35 studies, including 37 RCTs involving 8513 patients, to generate more comprehensive and reliable results.

The valuable findings of this network meta-analysis were as follows: (a) fluconazole, itraconazole, posaconazole, voriconazole, amphotericin $\mathrm{B}$, and caspofungin, but not micafungin, had the potential of reducing the incidence of proven and probable IFIs; (b) posaconazole was superior to fluconazole, itraconazole, and amphotericin $\mathrm{B}$ against proven and probable IFIs; (c) posaconazole was superior to fluconazole, itraconazole, voriconazole, amphotericin B, micafungin, and placebo against IArelated IFIs, and itraconazole had the potential of reducing IC-related IFIs; (d) posaconazole was superior to fluconazole, itraconazole, and amphotericin B in terms of all-cause mortality; (e) fluconazole, itraconazole, posaconazole, voriconazole, amphotericin $\mathrm{B}$, or caspofungin had the potential of reducing the risk of adverse events; (f) fluconazole, itraconazole, posaconazole, voriconazole, and amphotericin B were superior to micafungin in reducing the risk of adverse events, and fluconazole and voriconazole were superior to posaconazole and amphotericin B; $(\mathrm{g})$ all treatments were not different in terms of the need for empirical treatment and the proportion of successful treatment; and (h) posaconazole had the highest probability of being ranked the best against proven and probable IFIs.

To date, four topic-related meta-analyses $[3,4,16,17]$ included two traditional pairwise meta-analyses $[16,17]$ and two network meta-analyses [3, 4]. In 2002, Bow and colleagues [16] performed a meta-analysis of randomized controlled clinical trials to investigate the overall clinical efficacy of antifungal prophylaxis, including azole antifungal agents and low-dose intravenous amphotericin B, for severely neutropenic chemotherapy recipients. The aforementioned analysis included 38 eligible studies and showed that antifungal prophylaxis could reduce allcause mortality and IFI-related mortality. However, the efficacy and safety of individual antifungal prophylaxis agents were not investigated, thereby mitigating the reference value of the findings. On the contrary, the 


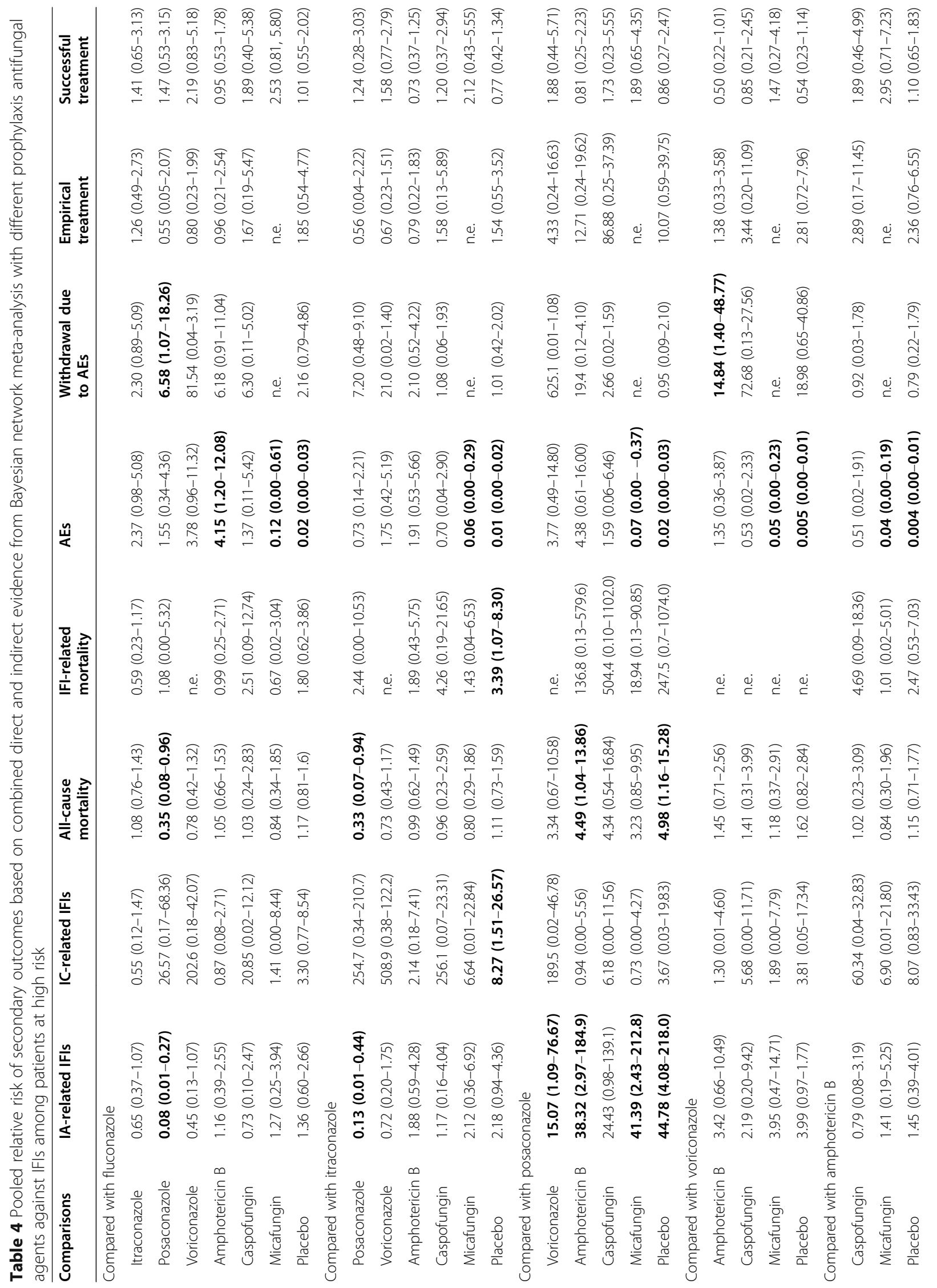




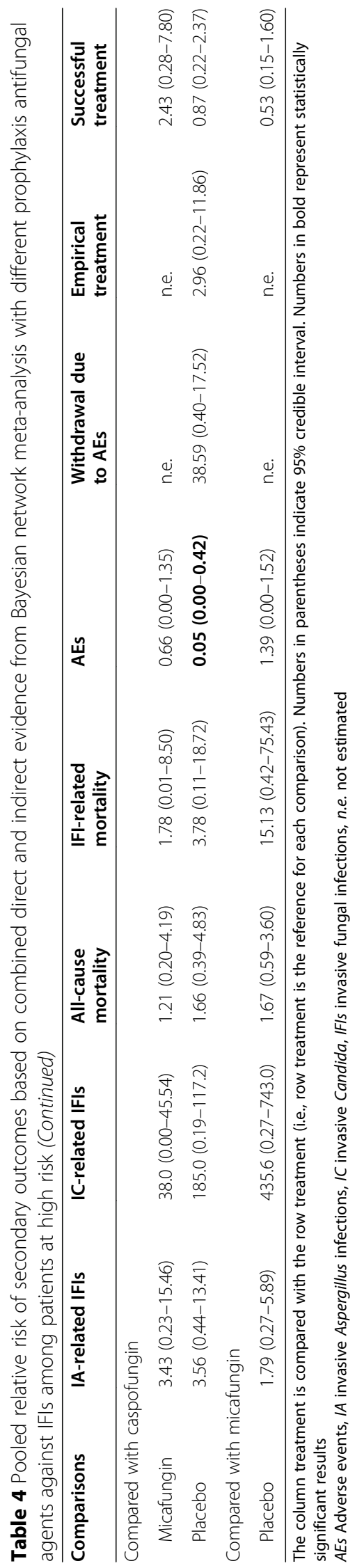






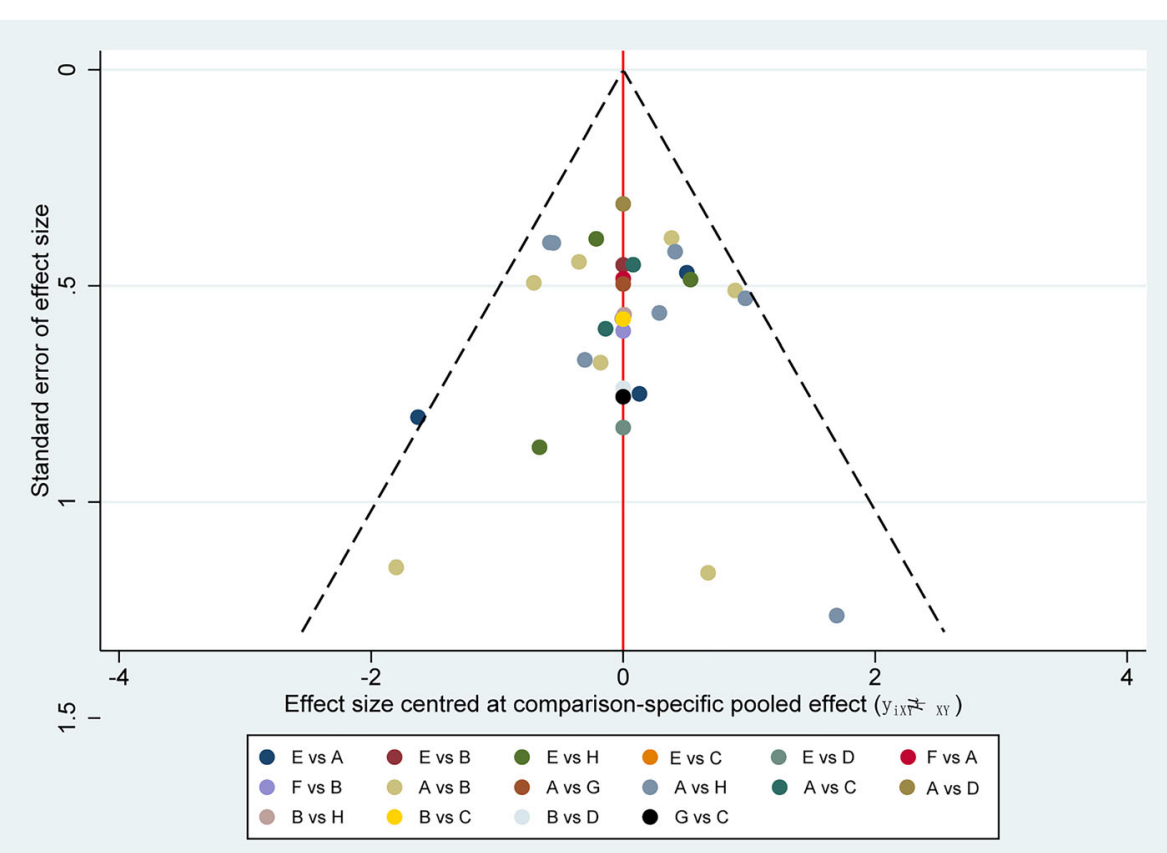

Fig. 5 Comparison-adjusted funnel plot for proven and probable IFIs. The vertical axis represents the standard error of effect size, and $x$ axis indicates effect size centered at comparison-specific pooled effect. Symmetrical funnel plot indicates the absence of publication bias. IFls, Invasive fungal infections; A, fluconazole; B, itraconazole; C, posaconazole; D, voriconazole; E, amphotericin B; F, caspofungin; G, micafungin; and H, placebo 
present analysis explored pure efficacy and safety of individual agents against IFIs and suggested that posaconazole was associated with the reduced incidence of allcause mortality. In 2005, Vardakas and colleagues [17] separately investigated the comparative efficacy of fluconazole versus itraconazole for antifungal prophylaxis in neutropenic patients with hematological malignancies. The pooled results based on five RCTs suggested that itraconazole was more effective than fluconazole in preventing IFIs in neutropenic patients with hematological malignancies; however, it was also associated with more adverse effects. The present analysis incorporated 35 studies involving $37 \mathrm{RCTs}$ to estimate the mixed efficacy of antifungal prophylaxis agents and found no significant difference between fluconazole and itraconazole in terms of the incidence of IFIs, mortality, and adverse events; the need for empirical treatment; and the proportion of successful treatment. In 2011, Freemantle et al. [4] compared between a systematic review and mixed treatment to investigate the potential of empirical, pre-emptive, and directed treatment strategies for invasive mold infections. This study suggested that caspofungin was superior to amphotericin $\mathrm{B}$ and voriconazole in the outcome of survival, and voriconazole was superior to amphotericin B for overall survival. However, the present study found no difference among caspofungin, amphotericin B, and voriconazole in terms of mortality. In 2016, Zhao and colleagues published a network metaanalysis [3] and found that all triazole antifungals were effective in preventing IFIs, which was consistent with the findings of the present analysis. Better than Zhao's network meta-analysis, the present analysis also suggested that amphotericin $B$ and caspofungin were effective against IFIs. Moreover, Zhao et al. found that posaconazole was more efficacious in reducing IFIs and all-cause death compared with fluconazole and itraconazole, which were also consistent with the findings of the present analysis.

The strength of this meta-analysis included the comprehensive and simultaneous assessment of the relative efficacy of all treatments against IFIs among patients at high risk. Given limited comparative effectiveness studies, it was difficult for patients and physicians to make informed decisions regarding which treatments were the most effective against IFIs. However, the meta-analysis had certain limitations related to both network analysis and individual studies, which merits further discussion. First, direct comparative effectiveness studies were scarce. Second, network meta-analyses might be susceptible to misinterpretation. The biggest threat to the validity of a network meta-analysis was conceptual heterogeneity involving considerable differences in participants, interventions, and specified regimes of targeted treatments, thus limiting the comparability of trials. It was assumed that patients enrolled in all included studies were sampled from the same theoretical population $[59,60]$. However, subtle differences were found in characteristics related to patients (adult patients, pediatric patients, patients receiving intensive chemotherapy for remission, and patients undergoing HCST), treatments (dose or form of individual treatment), and administration of agents (intravenous and oral). Third, ranking probabilities might be challenging to understand and did not always imply a clinically important difference. Hence, clinical decisions based on the findings should be made cautiously.

The individual studies included in the analysis also had some limitations, which also undermined the strength of the meta-analysis. Most of the studies focused on the efficacy against IFIs, with very few studies on mortality and adverse events, which limited the assessment of benefits of treatments, and hence a thorough assessment of risk-benefit profile could not be performed. Studies were also under the risk of detection bias with the suboptimal reporting of blinding of outcome assessors. Various study designs, including multicenter and single center, were used in different eligible studies. However, further sensitivity analysis or subgroup analysis was not designed based on the study design due to an insufficient number of eligible studies for the majority of comparisons. Therefore, it was critical to further investigate the impact of study design on pooled results when a sufficient number of eligible studies were published. Moreover, subgroup or sensitivity analysis was not designed according to the follow-up time due to an insufficient number of eligible studies for individual comparison. However, the time effects of treatments were investigated in individual studies, and no novel findings were reported $[13,51]$.

\section{Conclusions}

Despite these limitations, the present network metaanalysis provided a better understanding of the comparative efficacy of all potential treatments against IFIs among patients who were at high risk. Posaconazole might be a promising option against IFIs because it was superior to fluconazole, itraconazole, amphotericin B, voriconazole, or micafungin, although no significant difference was detected compared with caspofungin in terms of proven and probable IFIs and IA-related IFIs. Moreover, posaconazole also reduced all-cause mortality compared with fluconazole and itraconazole, and reduced the risk of adverse events compared with amphotericin B, fluconazole, itraconazole, posaconazole, voriconazole, amphotericin $B$, and caspofungin, although all treatments showed no significant difference in terms of the need for empirical treatment and the proportion of successful treatment. 


\section{Abbreviations}

IFIs: Invasive fungal infections; OR: Odds ratio; Crl: Credible interval; HSCT: Hematopoietic stem cell transplantation; RCTs: Randomized controlled trials; Cls: Confidence intervals; RR: Risk ratio

\section{Supplementary Information}

The online version contains supplementary material available at https://doi. org/10.1186/s12885-021-07973-8.

Additional file 1. PRISMA 2009 checklist

Additional file 2. Details of all targeted databases

Additional file 3: Fig. S1. Forest plot of possible and proven IFI. Fig. S2. Forest plot of IA-related possible and proven IFI. Fig. S3. Forest plot of IC-related possible and proven IFI. Fig. S4. Forest plot of all cause mortality. Fig. S5. Forest plot of IFl-related mortality. Fig. S6. Forest plot of $A E$. Fig. S7. Forest plot of withdrawal due AE. Fig. S8. Forest plot of empirical treatment. Fig. S9. Forest plot of successful treatment. Fig. S10. SUCRA of all drugs for proven and probable IFI

\section{Acknowledgments}

The authors express their sincere appreciation to all team members who performed the experiments in the present study.

\section{Authors' contributions}

HLZ and JC were involved in study conception and design. BY, BW, JW, and $J \mathrm{~L}$ participated in study selection and data extraction. ZMW, XYG, and CNW performed statistical analysis. HLZ and BY were involved in manuscript drafting. HLZ and JC were involved in manuscript revision. All authors approved the final manuscript for submission and publication.

\section{Funding}

Not applicable.

\section{Availability of data and materials}

All data generated or analyzed during this study are included in this published article [and its supplementary information files].

\section{Declarations}

Ethics approval and consent to participate

Not applicable.

\section{Consent for publication}

Not applicable.

\section{Competing interests}

The authors declare no competing interests.

\section{Author details}

${ }^{1}$ Department of Hematology, the First Affiliated Hospital of Jinan University, No.613 West Huangpu street, Guangzhou 510630, P. R. China. ${ }^{2}$ Emergency Department, the First Affiliated Hospital of Jinan University, No.613 West Huangpu street, Guangzhou 510630, P. R. China. ${ }^{3}$ Department of Urology Surgery, the First Affiliated Hospital of Jinan University, No.613 West Huangpu street, Guangzhou 510630, P. R. China.

Received: 15 November 2020 Accepted: 25 February 2021 Published online: 14 April 2021

\section{References}

1. Cornely OA, Leguay T, Maertens J, Vehreschild M, Anagnostopoulos A, Castagnola C, et al. Randomized comparison of liposomal amphotericin B versus placebo to prevent invasive mycoses in acute lymphoblastic leukaemia. J Antimicrob Chemother. 2017;72:2359-67.

2. Fisher BT, Zaoutis T, Dvorak CC, Nieder M, Zerr D, Wingard JR, et al. Effect of Caspofungin vs fluconazole prophylaxis on invasive fungal disease among children and young adults with acute myeloid leukemia: a randomized clinical trial. JAMA. 2019;322:1673-81.
3. Zhao YJ, Khoo AL, Tan G, Teng M, Tee C, Tan BH, et al. Network Metaanalysis and Pharmacoeconomic evaluation of fluconazole, Itraconazole Posaconazole, and Voriconazole in invasive fungal infection prophylaxis. Antimicrob Agents Chemother. 2016;60:376-86.

4. Freemantle N, Tharmanathan P, Herbrecht R. Systematic review and mixed treatment comparison of randomized evidence for empirical, pre-emptive and directed treatment strategies for invasive mould disease. J Antimicrob Chemother. 2011;66(Suppl 1):i25-35.

5. Garey KW, Rege M, Pai MP, Mingo DE, Suda KJ, Turpin RS, et al. Time to initiation of fluconazole therapy impacts mortality in patients with candidemia: a multi-institutional study. Clin Infect Dis. 2006;43:25-31.

6. Hale KA, Shaw PJ, Dalla-Pozza L, Maclntyre CR, Isaacs D, Sorrell TC. Epidemiology of paediatric invasive fungal infections and a case-control study of risk factors in acute leukaemia or post stem cell transplant. Br J Haematol. 2010;149:263-72.

7. Alexander S, Pole JD, Gibson P, Lee M, Hesser T, Chi SN, et al. Classification of treatment-related mortality in children with cancer: a systematic assessment. Lancet Oncol. 2015;16:e604-10.

8. Donnelly JP, Chen SC, Kauffman CA, Steinbach WJ, Baddley JW, Verweij PE, et al. Revision and Update of the Consensus Definitions of Invasive Fungal Disease From the European Organization for Research and Treatment of Cancer and the Mycoses Study Group Education and Research Consortium. Clin Infect Dis. 2019;6:1367-76.

9. Erjavec Z, Verweij PE. Recent progress in the diagnosis of fungal infections in the immunocompromised host. Drug Resist Updat. 2002;5:3-10.

10. Doan TN, Kirkpatrick CM, Walker P, Slavin MA, Ananda-Rajah MR, Morrissey $\mathrm{CO}$, et al. Primary antifungal prophylaxis in adult patients with acute lymphoblastic leukaemia: a multicentre audit. J Antimicrob Chemother. 2016;71:497-505.

11. Rüping MJ, Vehreschild JJ, Cornely OA. Patients at high risk of invasive fungal infections: when and how to treat. Drugs. 2008;68:1941-62.

12. Wingard JR, Carter SL, Walsh TJ, Kurtzberg J, Small TN, Baden LR, et al. Randomized, double-blind trial of fluconazole versus voriconazole for prevention of invasive fungal infection after allogeneic hematopoietic cell transplantation. Blood. 2010;116:5111-8.

13. Mattiuzzi GN, Cortes J, Alvarado G, Verstovsek S, Koller C, Pierce S, et al. Efficacy and safety of intravenous voriconazole and intravenous itraconazole for antifungal prophylaxis in patients with acute myelogenous leukemia or high-risk myelodysplastic syndrome. Support Care Cancer. 2011;19:19-26.

14. Park S, Kim K, Jang JH, Kim SJ, Kim WS, Chung DR, et al. Randomized trial of micafungin versus fluconazole as prophylaxis against invasive fungal infections in hematopoietic stem cell transplant recipients. J Inf Secur. 2016; 73:496-505.

15. Epstein DJ, Seo SK, Huang YT, Park JH, Klimek VM, Berman E, et al. Micafungin versus posaconazole prophylaxis in acute leukemia or myelodysplastic syndrome: a randomized study. J Inf Secur. 2018;77:227-34.

16. Bow EJ, Laverdière M, Lussier N, Rotstein C, Cheang MS, loannou S. Antifungal prophylaxis for severely neutropenic chemotherapy recipients: a meta analysis of randomized-controlled clinical trials. Cancer. 2002;94:3230-46.

17. Vardakas KZ, Michalopoulos A, Falagas ME. Fluconazole versus itraconazole for antifungal prophylaxis in neutropenic patients with haematological malignancies: a meta-analysis of randomised-controlled trials. $\mathrm{Br} J$ Haematol. 2005;131:22-8.

18. Moher D, Liberati A, Tetzlaff J, Altman DG, Group P. Preferred reporting items for systematic reviews and meta-analyses: the PRISMA statement. Ann Intern Med. 2009;151:264-9 W64.

19. Jansen JP, Fleurence R, Devine B, Itzler R, Barrett A, Hawkins N, et al. Interpreting indirect treatment comparisons and network meta-analysis for health-care decision making: report of the ISPOR task force on indirect treatment comparisons good research practices: part 1. Value Health. 2011; 14:417-28.

20. Higgins JP, Altman DG, Gotzsche PC, Juni P, Moher D, Oxman AD, et al. The Cochrane Collaboration's tool for assessing risk of bias in randomised trials. BMJ. 2011;343:d5928.

21. DerSimonian R, Laird N. Meta-analysis in clinical trials. Control Clin Trials. 1986;7:177-88.

22. Higgins JP, Thompson SG, Deeks JJ, Altman DG. Measuring inconsistency in meta-analyses. BMJ. 2003;327:557-60.

23. Higgins JPT, Altman DG, Sterne JAC. In: JPT H, editor. Cochrane handbook for systematic reviews of interventions: In, The Cochrane collaboration; 2011. Available from http://www.cochrane-handbook.org. . 
24. Page MJ, McKenzie JE, Higgins JPT. Tools for assessing risk of reporting biases in studies and syntheses of studies: a systematic review. BMJ Open. 2018;8:e019703.

25. Dias S, Sutton AJ, Ades AE, Welton NJ. Evidence synthesis for decision making 2: a generalized linear modeling framework for pairwise and network meta-analysis of randomized controlled trials. Med Decis Mak. 2013;33:607-17

26. Lu G, Ades AE. Combination of direct and indirect evidence in mixed treatment comparisons. Stat Med. 2004;23:3105-24

27. Sutton A, Ades AE, Cooper N, Abrams K. Use of indirect and mixed treatment comparisons for technology assessment. PharmacoEconomics. 2008;26:753-67.

28. Song G-M, Liu X-L, Bian W, Wu J, Deng Y-H, Zhang H, et al. Systematic review with network meta-analysis: comparative efficacy of different enteral immunonutrition formulas in patients underwent gastrectomy. Oncotarget. 2017:8:23376-88.

29. Chaimani A, Higgins JP, Mavridis D, Spyridonos P, Salanti G. Graphical tools for network meta-analysis in STATA. PLoS One. 2013;8:e76654.

30. Singh S, Murad MH, Chandar AK, Bongiorno CM, Singal AK, Atkinson SR, et al. Comparative Effectiveness of Pharmacological Interventions for Severe Alcoholic Hepatitis: A Systematic Review and Network Meta-analysis. Gastroenterology. 2015;149:958-70.e12.

31. Winston DJ, Chandrasekar PH, Lazarus HM, Goodman JL, Silber JL, Horowitz $\mathrm{H}$, et al. Fluconazole prophylaxis of fungal infections in patients with acute leukemia. Results of a randomized placebo-controlled, double-blind, multicenter trial. Ann Intern Med. 1993;118:495-503.

32. Chandrasekar PH, Gatny CM. Effect of fluconazole prophylaxis on fever and use of amphotericin in neutropenic cancer patients. Bone Marrow Transplantation Team. Chemotherapy. 1994;40:136-43.

33. Annaloro C, Oriana A, Tagliaferri E, Bertolli V, Della Volpe A, Soligo D, et al. Efficacy of different prophylactic antifungal regimens in bone marrow transplantation. Haematologica. 1995;80:512-7.

34. Ellis ME, Halim MA, Spence D, Ernst P, Clink H, Kalin M, et al. Systemic amphotericin $B$ versus fluconazole in the management of antibiotic resistant neutropenic fever--preliminary observations from a pilot, exploratory study. J Inf Secur. 1995;30:141-6.

35. Schaffner A, Schaffner M. Effect of prophylactic fluconazole on the frequency of fungal infections, amphotericin B use, and health care costs in patients undergoing intensive chemotherapy for hematologic neoplasias. J Infect Dis. 1995:172:1035-41.

36. Slavin MA, Osborne B, Adams R, Levenstein MJ, Schoch HG, Feldman AR, et al. Efficacy and safety of fluconazole prophylaxis for fungal infections after marrow transplantation--a prospective, randomized, double-blind study. J Infect Dis. 1995;171:1545-52.

37. Yamaç K, Senol E, Haznedar R. Prophylactic use of fluconazole in neutropenic cancer patients. Postgrad Med J. 1995;71:284-6.

38. Menichetti F, Del Favero A, Martino P, Bucaneve G, Micozzi A, Girmenia C, et al. Itraconazole oral solution as prophylaxis for fungal infections in neutropenic patients with hematologic malignancies: a randomized, placebo-controlled, double-blind, multicenter trial. GIMEMA infection program. Gruppo Italiano Malattie Ematologiche dell' Adulto. Clin Infect Dis. 1999;28:250-5.

39. Rotstein C, Bow EJ, Laverdiere M, loannou S, Carr D, Moghaddam N. Randomized placebo-controlled trial of fluconazole prophylaxis for neutropenic cancer patients: benefit based on purpose and intensity of cytotoxic therapy. The Canadian fluconazole prophylaxis study group. Clin Infect Dis. 1999;28:331-40.

40. Harousseau JL, Dekker AW, Stamatoullas-Bastard A, Fassas A, Linkesch W, Gouveia J, et al. Itraconazole oral solution for primary prophylaxis of fungal infections in patients with hematological malignancy and profound neutropenia: a randomized, double-blind, double-placebo, multicenter trial comparing itraconazole and amphotericin B. Antimicrob Agents Chemother. 2000;44:1887-93.

41. Karthaus M, Doellmann T, Klimasch T, Elser C, Rosenthal C, Ganser A, et al Intensive intravenous amphotericin $B$ for prophylaxis of systemic fungal infections. Results of a prospective controlled pilot study in acute leukemia patients. Chemotherapy. 2000;46:293-302.

42. Laverdière $M$, Rotstein C, Bow EJ, Roberts RS, loannou S, Carr D, et al. Impact of fluconazole prophylaxis on fungal colonization and infection rates in neutropenic patients. The Canadian fluconazole study. J Antimicrob Chemother. 2000;46:1001-8.
43. Nucci M, Biasoli I, Akiti T, Silveira F, Solza C, Barreiros G, et al. A double-blind randomized, placebo-controlled trial of itraconazole capsules as antifungal prophylaxis for neutropenic patients. Clin Infect Dis. 2000;30:300-5.

44. Wolff SN, Fay J, Stevens D, Herzig RH, Pohlman B, Bolwell B, et al. Fluconazole vs low-dose amphotericin $B$ for the prevention of fungal infections in patients undergoing bone marrow transplantation: a study of the north American marrow transplant group. Bone Marrow Transplant. 2000;25:853-9.

45. Cornely OA, Maertens J, Winston DJ, Perfect J, Ullmann AJ, Walsh TJ, et al. Posaconazole vs. fluconazole or itraconazole prophylaxis in patients with neutropenia. N Engl J Med. 2007;356:348-59.

46. Glasmacher A, Cornely O, Ullmann AJ, Wedding U, Bodenstein H, Wandt $H$, et al. An open-label randomized trial comparing itraconazole oral solution with fluconazole oral solution for primary prophylaxis of fungal infections in patients with haematological malignancy and profound neutropenia. J Antimicrob Chemother. 2006;57:317-25.

47. Ito Y, Ohyashiki K, Yoshida I, Takeuchi M, Aoyama Y, Mugitani A, et al. The prophylactic effect of itraconazole capsules and fluconazole capsules for systemic fungal infections in patients with acute myeloid leukemia and myelodysplastic syndromes: a Japanese multicenter randomized, controlled study. Int J Hematol. 2007;85:121-7.

48. Marks DI, Pagliuca A, Kibbler CC, Glasmacher A, Heussel CP, Kantecki M, et al. Voriconazole versus itraconazole for antifungal prophylaxis following allogeneic haematopoietic stem-cell transplantation. Br J Haematol. 2011; 155:318-27.

49. Mike H. Prevention of Invasive Fungal Infections (IFIs) in Subjects Receiving Chemotherapy for Acute Lymphoblastic Leukemia. https://clinicaltrialsgov/ show/NCT01259713. 2010.

50. Shen Y, Huang XJ, Wang JX, Jin J, Hu JD, Yu K, et al. Posaconazole vs. fluconazole as invasive fungal infection prophylaxis in China: a multicenter, randomized, open-label study. Int J Clin Pharmacol Ther. 2013;51:738-45.

51. Mattiuzzi GN, Alvarado G, Giles FJ, Ostrosky-Zeichner L, Cortes J, O'Brien S, et al. Open-label, randomized comparison of itraconazole versus caspofungin for prophylaxis in patients with hematologic malignancies. Antimicrob Agents Chemother. 2006;50:143-7.

52. Gloria NM. Liposomal Amphotericin B (Ambisome) Versus Oral Voriconazole for the Prevention of Invasive Fungal Infections. https://clinicaltrialsgov/ show/NCT00418951. 2007.

53. Chaftari AM, Hachem RY, Ramos E, Kassis C, Campo M, Jiang Y, et al. Comparison of posaconazole versus weekly amphotericin B lipid complex for the prevention of invasive fungal infections in hematopoietic stem-cell transplantation. Transplantation. 2012;94:302-8.

54. Marr KA, Crippa F, Leisenring W, Hoyle M, Boeckh M, Balajee SA, et al. Itraconazole versus fluconazole for prevention of fungal infections in patients receiving allogeneic stem cell transplants. Blood. 2004;103:1527-33.

55. Oren I, Rowe JM, Sprecher H, Tamir A, Benyamini N, Akria L, et al. A prospective randomized trial of itraconazole vs fluconazole for the prevention of fungal infections in patients with acute leukemia and hematopoietic stem cell transplant recipients. Bone Marrow Transplant. 2006;38:127-34.

56. Mandhaniya S, Swaroop C, Thulkar S, Vishnubhatla S, Kabra SK, Xess I, et al. Oral voriconazole versus intravenous low dose amphotericin B for primary antifungal prophylaxis in pediatric acute leukemia induction: a prospective, randomized, clinical study. J Pediatr Hematol Oncol. 2011;33:e333-41.

57. Mattiuzzi GN, Estey E, Raad I, Giles F, Cortes J, Shen Y, et al. Liposomal amphotericin $B$ versus the combination of fluconazole and itraconazole as prophylaxis for invasive fungal infections during induction chemotherapy for patients with acute myelogenous leukemia and myelodysplastic syndrome. Cancer. 2003;97:450-6.

58. Vehreschild JJ, Böhme A, Buchheidt D, Arenz D, Harnischmacher U, Heussel $C P$, et al. A double-blind trial on prophylactic voriconazole (VRC) or placebo during induction chemotherapy for acute myelogenous leukaemia (AML). J Inf Secur. 2007:55:445-9.

59. Cipriani A, Higgins JP, Geddes JR, Salanti G. Conceptual and technical challenges in network meta-analysis. Ann Intern Med. 2013;159:130-7.

60. Puhan MA, Schunemann HJ, Murad MH, Li T, Brignardello-Petersen R, Singh JA, et al. A GRADE working group approach for rating the quality of treatment effect estimates from network meta-analysis. BMJ. 2014;349:95630.

\section{Publisher's Note}

Springer Nature remains neutral with regard to jurisdictional claims in published maps and institutional affiliations. 